\title{
SUPPLEMENTARY MATERIALS: ON THE CONDITIONAL DISTRIBUTIONS AND THE EFFICIENT SIMULATIONS OF EXPONENTIAL INTEGRALS OF GAUSSIAN RANDOM FIELDS
}

\author{
By Jingchen LiU*, And Gonguun Xu
}

Columbia University and University of Minnesota

Proof of Proposition 14. The proof of this proposition basically replicates that of Theorem 3.4 in Liu and $\mathrm{Xu}$ (2012) under slightly bigger remainder terms of the Taylor expansions. Therefore, we only layout the key steps. We consider the following change of measure

$$
\frac{d Q_{2}}{d P} \triangleq \int_{T} \frac{1}{m e s(T)} L R_{2}(t) d t
$$

where $L R_{2}$ is defined in (3.11). Define another set

$$
\mathcal{L}_{1}=\left\{|f(\tau)-u|<u^{1 / 2+\epsilon},|y|<u^{1 / 2+\epsilon},|z|<u^{1 / 2+\epsilon}, \sup _{|t-\tau|<u^{-1+\delta}}|g(t)|<u^{-1-\delta}\right\} .
$$

With a similar proof as in Lemma 17, we have that

$$
P\left(\mathcal{I}(T)>b, \mathcal{L}_{1}^{c}\right)=E^{Q_{2}}\left[\frac{d P}{d Q_{2}} ; \mathcal{I}(T)>b, \mathcal{L}_{1}^{c}\right]=o(1) v(b) .
$$

In what follows, we approximate $E^{Q_{2}}\left[d P / d Q_{2} ; \mathcal{I}(T)>b, \mathcal{L}_{1}\right]$. Note that for $\tau$ uniformly distributed on $T$,

$$
\begin{aligned}
P(\mathcal{I}(T)>b) & =E^{Q_{2}}\left[\frac{d P}{d Q_{2}} ; \mathcal{I}(T)>b, \mathcal{L}_{1}\right] \\
& =\int_{T} E^{Q_{2}}\left[\left(\int_{T} e^{-\frac{1}{2} u_{t}^{2}+\left[w_{t}+u_{\tau} C(t-\tau)\right] u_{t}} d t\right)^{-1} ; \mathcal{I}(T)>b, \mathcal{L}_{1} \mid \tau\right] d \tau .
\end{aligned}
$$

For a given $\delta^{\prime}>0$ small enough, we consider two cases for $\tau$ : first, $\left\{t:|t-\tau| \leq u^{-1 / 2+\delta^{\prime}}\right\} \subset$ $T$ and otherwise. For the first situation, the derivation for the above expectation takes a similar three-step procedure as that in the proof of Theorem 3 . We only state the key steps here.

Part 1. On the set $\mathcal{L}_{1}$ and for a given $\tau$, following Part 1 in the proof of Theorem 3 , in particular results in (4.20), we have $\mathcal{I}(T)>b$ if and only if

$$
\mathcal{A}^{\prime}>u^{-1} \sigma^{-1} \xi_{u}
$$

where

$\mathcal{A}^{\prime}=w+\frac{1}{2} \tilde{y}^{\top}(u I-\tilde{\mathbf{z}})^{-1} \tilde{y}-\frac{1}{2 \sigma} \log \operatorname{det}\left(I-u^{-1} \tilde{\mathbf{z}}\right)+\frac{1}{8 \sigma^{2} u} \sum_{i} \partial_{i i i i}^{4} C(0)+u^{-1-\delta_{0} / 4} o(|w|+|y|+|z|)$.

${ }^{*}$ This research is supported by Institute of Education Sciences, through Grant R305D100017, NSF CMMI-1069064, and NSF SES-1323977. 
Part 2. Let $\lambda_{u}=u^{-1 / 2+\delta}, \epsilon<\delta<\delta^{\prime}$. We first split the integral into two parts, that is,

$$
\begin{aligned}
\int_{T} e^{-\frac{1}{2} u_{t}^{2}+\left[w_{t}+u_{\tau} C(t-\tau)\right] u_{t}} d t & =\int_{T} e^{-u^{2} / 2+u_{t}\left[w_{t}+u_{\tau} C(t-\tau)+\mu_{\sigma}(t)\right]+\frac{1}{2} \mu_{\sigma}^{2}(t)} d t \\
& =\int_{|t-\tau|<\lambda_{u}} \cdots+\int_{|t-\tau|>\lambda_{u}} \cdots \\
& =I_{2}+I_{3} .
\end{aligned}
$$

For $I_{2}$ term, note that $|t-\tau| \leq \lambda_{u}=u^{-1 / 2+\delta}$. We insert the Taylor expansion of $\mu_{\sigma}(t)$

$$
I_{2}=(1+o(1)) e^{u^{2} / 2-u \mu_{\sigma}(\tau)+\mu_{\sigma}^{2}(\tau) / 2} \int_{|t-\tau|<\lambda_{u}} e^{u_{t}\left[w_{t}+u_{\tau} C(t-\tau)-u+\mu_{\sigma}(\tau)\right]} d t .
$$

Similar to Part 1, we insert the Taylor expansion of $w_{t}$ and $C(t-\tau)$ to the above display and the integral equals $\left(\zeta_{u}=O\left(u^{-1 / 2+\delta}\right)\right)$

$$
\begin{aligned}
& \int_{|t-\tau|<\lambda_{u}} e^{u_{t}\left[w_{t}+u_{\tau} C(t-\tau)-u+\mu_{\sigma}(\tau)\right]} d t \\
= & (1+o(1)) \exp \left\{\left(u_{\tau}+\zeta_{u}\right)\left[w+\frac{1}{2} y\left(u_{\tau} I-\mathbf{z}\right)^{-1} y\right]\right\} \\
& \times \int_{|t-\tau|<\lambda_{u}} \exp \left\{( u _ { \tau } + \zeta _ { u } ) \left[-\frac{1}{2}\left(t-\tau-\left(u_{\tau} I-\mathbf{z}\right)^{-1} y\right)^{\top}\left(u_{\tau} I-\mathbf{z}\right)\left(t-\tau-\left(u_{\tau} I-\mathbf{z}\right)^{-1} y\right)\right.\right. \\
& \left.\left.+u_{\tau} C_{4}(t-\tau)+g(t-\tau)+R(t-\tau)\right]\right\} d t .
\end{aligned}
$$

We consider the change of variable that $s=\left(u+\zeta_{u}\right)^{1 / 2}\left(u_{\tau} I-\tilde{\mathbf{z}}\right)^{1 / 2}(t-\tau)$ and obtain the following approximation via a similar argument as in the part 2.1 of proof of Theorem 3 .

$$
\begin{aligned}
I_{2}= & (1+o(1)) \kappa u^{-d} e^{u^{2} / 2-u \mu_{\sigma}(\tau)+\frac{1}{2} \mu_{\sigma}^{2}(\tau)-\frac{1}{8 \sigma^{2}} \sum_{i} \partial_{i i i i}^{4} C(0)} \\
& \times \exp \left\{u_{\tau} \mathcal{A}^{\prime}-\frac{1}{2 \sigma} \operatorname{Tr}\left(\mathbf{z}+\mu_{\sigma}(\tau) I+\Delta \mu_{\sigma}(\tau)\right)-y^{\top} \partial \mu_{\sigma}(\tau)-\frac{1}{2}\left|\partial \mu_{\sigma}(\tau)\right|^{2}\right. \\
& \left.+u^{-\delta_{0} / 2} O\left(w+|y|^{2}+|z|^{2}\right)\right\}
\end{aligned}
$$

where

$$
\begin{aligned}
\kappa= & \int_{\left|u_{\tau}-1 / 2\left(u_{\tau} I-\mathbf{z}\right)^{-1 / 2} s\right|<\lambda_{u}} \exp \left\{-\frac{1}{2}\left|s-\left(u+\zeta_{u}\right)^{1 / 2}\left(u_{\tau} I-\mathbf{z}\right)^{-1 / 2} y\right|^{2}\right\} d s \\
& \times E\left[\exp \left\{\left(\tilde{u}+\zeta_{u}\right) g\left(\left(\tilde{u}+\zeta_{u}\right)^{-1 / 2}(\tilde{u} I-\tilde{\mathbf{z}})^{-1 / 2} S^{\prime}\right)\right\}\right] .
\end{aligned}
$$

Here $S^{\prime}$ is a random variable taking values on the set $\left\{s:\left|u_{\tau}{ }^{-1 / 2}\left(u_{\tau} I-\mathbf{z}\right)^{-1 / 2} s\right|<\lambda_{u}\right\}$ with density proportional to

$e^{-\frac{1}{2}\left|s-\left(u+\zeta_{u}\right)^{1 / 2}\left(u_{\tau} I-\mathbf{Z}\right)^{-1 / 2} y\right|^{2}+u_{\tau}\left(u+\zeta_{u}\right) C_{4}\left(\left(u+\zeta_{u}\right)^{-1 / 2}\left(u_{\tau} I-\mathbf{Z}\right)^{-1 / 2} s\right)+\left(u+\zeta_{u}\right) R\left(\left(u+\zeta_{u}\right)^{-1 / 2}\left(u_{\tau} I-\mathbf{Z}\right)^{-1 / 2} s\right) .}$

Thanks to Lemma 5.5 in Liu and $\mathrm{Xu}(2012), I_{3}$ is of a much smaller order than $I_{2}$ and we obtain the following result:

$$
\begin{aligned}
I_{2}+I_{3}= & (1+o(1))\left(\kappa+O\left(e^{-\delta^{*} u^{1+2 \delta}} e^{u \sup |g(t)|}\right)\right) u^{-d} e^{\frac{u^{2}}{2}-u \mu_{\sigma}(\tau)+\frac{1}{2} \mu_{\sigma}^{2}(\tau)-\frac{\sum_{i} \partial_{i i i i}^{4} C(0)}{8 \sigma^{2}}} \\
& \times \exp \left\{u_{\tau} \mathcal{A}^{\prime}-\frac{1}{2 \sigma} \operatorname{Tr}\left(\mathbf{z}+\mu_{\sigma}(\tau) I+\Delta \mu_{\sigma}(\tau)\right)-y^{\top} \partial \mu_{\sigma}(\tau)-\frac{1}{2}\left|\partial \mu_{\sigma}(\tau)\right|^{2}\right. \\
& \left.+u^{-\delta_{0} / 2} O\left(w+|y|^{2}+|z|^{2}\right)\right\}
\end{aligned}
$$


Part 3. We now put together the results from Part 1 and Part 2 and obtain an approximation of $E^{Q_{2}}\left[d P / d Q_{2} ; \mathcal{I}(T)>b, \mathcal{L}_{1}\right]$. This part is completely analogous to Part 3 of the proof of Theorem 3 .

$$
\begin{aligned}
& E^{Q_{2}}\left[\frac{d P}{d Q_{2}} ; \sigma u \mathcal{A}^{\prime}>\xi_{u}, \mathcal{L}_{1} \mid \tau\right] \\
= & \operatorname{mes}(T) E^{Q_{2}}\left[\frac{1}{I_{2}+I_{3}} ; \sigma u \mathcal{A}^{\prime}>\xi_{u}, \mathcal{L}_{1} \mid \tau\right] \\
= & \operatorname{mes}(T) \int_{\mathcal{L}} E\left[\frac{1}{I_{2}+I_{3}} ; \sigma u \mathcal{A}^{\prime}>\xi_{u} \mid \tau, w, y, z\right] h(w, y, z) d w d y d z,
\end{aligned}
$$

where $h$ is the density function of $\left(f(\tau), \partial f(\tau), \partial^{2} f(\tau)\right)$ under the measure $P$. Plugging in $(0.2)$ and $h(w, y, z)$, a similar derivation as in Part 3 in the proof of Theorem 3 yields that $\operatorname{mes}(T)^{-1} E^{Q_{2}}\left[d P / d Q_{2} ; \mathcal{I}(T)>b, \mathcal{L}_{1} \mid \tau\right]$ equals

$$
\begin{aligned}
& (1+o(1)) u^{d-1} e^{-\frac{1}{2}\left(u-\mu_{\sigma}(\tau)\right)^{2}+\frac{1}{2 \sigma} \operatorname{Tr}\left(\mu_{\sigma}(\tau) I+\Delta \mu_{\sigma}(\tau)\right)+\left|\partial \mu_{\sigma}(\tau)\right|^{2}+\frac{1^{\top} \mu_{22} 1}{8 \sigma^{2}}+\frac{1}{8 \sigma^{2}} \sum_{i} \partial_{i i i i}^{4} C(0)} \\
& \frac{|\Gamma|^{-1 / 2}}{(2 \pi)^{\frac{(d+1)(d+2)}{4}}} \int_{\tilde{z} \in R^{d(d+1) / 2}} \exp \left\{-\frac{1}{2}\left[\frac{\left|\mu_{20} \mu_{22}^{-1} \tilde{z}\right|^{2}}{1-\mu_{20} \mu_{22}^{-1} \mu_{02}}+\left|\mu_{22}^{-1 / 2} \tilde{z}-\mu_{22}^{1 / 2} \frac{\mathbf{1}}{2 \sigma}\right|^{2}\right]\right\} d \tilde{z} .
\end{aligned}
$$

Note that $\tau$ is uniformly distributed over $T$ and thus we conclude the situation when $\tau$ is at least $u^{-1 / 2+\delta^{\prime}}$ away from the boundary.

For the case in which $\tau$ is within $u^{-1 / 2+\delta^{\prime}}$ distance from the boundary of $T$, we need to follow a similar derivation as the proof of Theorem $3.4 \mathrm{in} \mathrm{Liu} \mathrm{and} \mathrm{Xu}$ (2012). We do not provide the details. An intuitive interpretation is that the important region of the integral $\int e^{f(t)} d t$ might be cut off by the boundary of $T$. Therefore, in cases that $\tau$ is too close to the boundary, the tail $\int e^{f(t)} d t$ is not heavier than that of the interior case. In fact, for the purpose of proving our main theorem, we only need to establish a lower bound of $v(b)$, thus, the boundary case can be in fact omitted. Thereby, we conclude the proof.

Proof of Lemma 17. Define another set

$$
\mathcal{L}_{1}=\left\{|f(\tau)-u|<u^{1 / 2+\epsilon},|y|<u^{1 / 2+\epsilon},|z|<u^{1 / 2+\epsilon}, \sup _{|t-\tau|<u^{-1+\delta}}|g(t)|<u^{-1-\delta}\right\} .
$$

We first show that

$$
E^{Q}\left[\left(\frac{d P}{d Q}\right)^{2} ; \int_{T} e^{\mu(t)+\sigma f(t)} d t>b, \mathcal{L}_{1}^{c}\right]=o(1) v(b)^{2}
$$

In this proof, we mainly use the last component of the mixture $L R_{2}(t)$ that has not been used in the main proof of the theorem. Note that $d Q / d P \geq \rho_{2} \int_{T} L R_{2}(t) / \operatorname{mes}(T) d t$ and therefore

$$
\begin{aligned}
& E^{Q}\left[\left(\frac{d P}{d Q}\right)^{2} ; \int_{T} e^{\mu(t)+\sigma f(t)} d t>b, \mathcal{L}_{1}^{c}\right] \\
\leq & E^{Q}\left[\left(\rho_{2} \int_{T} \frac{1}{m e s(T)} L R_{2}(t) d t\right)^{-2} ; \mathcal{I}(T)>b, \mathcal{L}_{1}^{c}\right] \\
= & \frac{1}{\rho_{2}^{2}} E^{Q}\left[\left(\frac{1}{m e s(T)} \int_{T} e^{-\frac{1}{2} u_{t}^{2}+u_{t} f(t)} d t\right)^{-2} ; \frac{\mathcal{I}(T)}{\operatorname{mes}(T)}>\frac{b}{m e s(T)}, \mathcal{L}_{1}^{c}\right] .
\end{aligned}
$$


On the set

$$
\frac{1}{m e s(T)} \int_{T} e^{\sigma\left(\mu_{\sigma}(t)+f(t)\right)} d t=\frac{\mathcal{I}(T)}{m e s(T)}>\frac{b}{m e s(T)}
$$

we have for large $b$,

$$
\begin{aligned}
& \frac{1}{m e s(T)} \int_{T} e^{-\frac{1}{2} u_{t}^{2}+u_{t} f(t)} d t \\
= & e^{-\frac{1}{2} u^{2}} \times \frac{1}{\operatorname{mes}(T)} \int_{T} e^{\left(u-\mu_{\sigma}(t)\right) \cdot\left(f(t)+\mu_{\sigma}(t)\right)+\frac{1}{2} \mu_{\sigma}^{2}(t)} d t \\
\geq & e^{-\frac{1}{2} u^{2}+\frac{1}{2} \min _{t \in T} \mu_{\sigma}^{2}(t)} \times \frac{1}{\operatorname{mes}(T)} \times\left[\int_{T \cap\left\{f(t)+\mu_{\sigma}(t) \geq 0\right\}} e^{\left(u-\max _{t \in T} \mu_{\sigma}(t)\right)\left(f(t)+\mu_{\sigma}(t)\right)} d t\right. \\
& \left.+\int_{T \cap\left\{f(t)+\mu_{\sigma}(t)<0\right\}} e^{\left(u-\min _{t \in T} \mu_{\sigma}(t)\right)\left(f(t)+\mu_{\sigma}(t)\right)} d t\right] \\
\geq & \frac{1}{2} e^{-\frac{1}{2} u^{2}+\frac{1}{2} \min _{t \in T} \mu_{\sigma}^{2}(t)} \times\left[\frac{1}{\operatorname{mes}(T)} \int_{T} e^{\left(u-\max _{t \in T} \mu_{\sigma}(t)\right)\left(f(t)+\mu_{\sigma}(t)\right)} d t\right]
\end{aligned}
$$

We apply Jensen's inequality to the term in the bracket and have that

$$
\begin{aligned}
& \geq \frac{1}{2} e^{-\frac{1}{2} u^{2}+\frac{1}{2} \min _{t \in T} \mu_{\sigma}^{2}(t)} \times\left[\frac{1}{m e s(T)} b\right]^{\left(u-\max _{t \in T} \mu_{\sigma}(t)\right) / \sigma} \\
& \geq e^{\frac{1}{2} u^{2}-c_{1} u \log u}
\end{aligned}
$$

where $c_{1}$ is some constant. The last step of the above display uses the fact that $u-\log b=$ $O(\log u)$. Therefore, the above derivation implies that

$$
(0.4) \leq \rho_{2}^{-2} e^{-u^{2}+2 c_{1} u \log u} \times Q\left(\mathcal{L}_{1}^{c}\right)
$$

By the Borel-TIS Lemma (Lemma 16), we have that $Q\left(\mathcal{L}_{1}^{c}\right)=O(1) e^{-\omega u^{1+2 \epsilon}}$ for some $\omega>0$ and thus

$$
E^{Q}\left[\left(\frac{d P}{d Q}\right)^{2} ; \mathcal{I}(T)>b, \mathcal{L}_{1}^{c}\right]=o(1) \rho_{2}^{-2} e^{-u^{2}-\frac{\omega}{2} u^{1+2 \epsilon}}=o(1) v^{2}(b) .
$$

We now proceed to bound for the rest of the expectation, that is,

$$
E^{Q}\left[\left(\frac{d P}{d Q}\right)^{2} ; \mathcal{I}(T)>b, \mathcal{L}^{c} \cap \mathcal{L}_{1}\right]
$$

This requires some fine analysis. Similar to the proof on the set $\mathcal{L}_{1}$, we still focus on the last component of the likelihood ratio corresponding to $L R_{2}$. Let

$$
\mathcal{L}_{2}=\mathcal{L}^{c} \cap \mathcal{L}_{1}
$$

Since $\mathcal{L}_{2} \subset \mathcal{L}_{1}$, the rest of the derivations are on the set $\mathcal{L}_{1}$. Note that

$$
E^{Q}\left[\left(\frac{d P}{d Q}\right)^{2} ; \mathcal{I}(T)>b, \mathcal{L}_{2}\right] \leq \operatorname{mes}^{2}(T) \rho_{2}^{-2} E^{Q}\left[\left(\int_{T} e^{-\frac{1}{2} u_{t}^{2}+\left[w_{t}+u_{\tau} C(t-\tau)\right] u_{t}} d t\right)^{-2} ; \mathcal{I}(T)>b, \mathcal{L}_{2}\right] .
$$

The derivation for the above expectation takes a similar three-step procedure as that in the proof of Theorem 3. We only state the key steps here. 
Part 1. On the set $\mathcal{L}_{1}$ and for a given $\tau$, following Part 1 in the proof of Theorem 3 , in particular results in (4.20), we have $\mathcal{I}(T)>b$ if and only if

$$
\mathcal{A}^{\prime}>u^{-1} \sigma^{-1} \xi_{u}
$$

where $\mathcal{A}^{\prime}=w+\frac{1}{2} \tilde{y}^{\top}(u I-\tilde{\mathbf{z}})^{-1} \tilde{y}-\frac{1}{2 \sigma} \log \operatorname{det}\left(I-u^{-1} \tilde{\mathbf{z}}\right)+\frac{1}{8 \sigma^{2} u} \sum_{i} \partial_{i i i i}^{4} C(0)+u^{-1} o(|w|+$ $|y|+|z|)$.

Part 2. Let $\lambda_{u}=u^{-1 / 2+\delta}$ and we have that

$$
\begin{aligned}
& \int_{T} e^{-\frac{1}{2} u_{t}^{2}+\left[w_{t}+u_{\tau} C(t-\tau)\right] u_{t}} d t \\
\geq & e^{-u^{2} / 2} \int_{|t-\tau|<\lambda_{u}} e^{u_{t}\left[w_{t}+u_{\tau} C(t-\tau)+\mu_{\sigma}(t)\right]+\frac{1}{2} \mu_{\sigma}^{2}(t)} d t \\
= & (1+o(1)) e^{u^{2} / 2-u \mu_{\sigma}(\tau)+\mu_{\sigma}^{2}(\tau) / 2} \int_{|t-\tau|<\lambda_{u}} e^{u_{t}\left[w_{t}+u_{\tau} C(t-\tau)-u+\mu_{\sigma}(\tau)\right]} d t .
\end{aligned}
$$

Similar to Part 1, we insert the Taylor expansion of $w_{t}$ and $C(t-\tau)$ to the above display and the integral equals $\left(\zeta_{u}=O\left(u^{-1 / 2+\delta}\right)\right)$

$$
\begin{gathered}
\int_{|t-\tau|<\lambda_{u}} e^{u_{t}\left[w_{t}+u_{\tau} C(t-\tau)-u+\mu_{\sigma}(\tau)\right]} d t \\
=(1+o(1)) \exp \left\{\left(u_{\tau}+\zeta_{u}\right)\left[w+\frac{1}{2} y\left(u_{\tau} I-\mathbf{z}\right)^{-1} y\right]\right\} \\
\times \int_{|t-\tau|<\lambda_{u}} \exp \left\{( u _ { \tau } + \zeta _ { u } ) \left[-\frac{1}{2}\left(t-\tau-\left(u_{\tau} I-\mathbf{z}\right)^{-1} y\right)^{\top}\left(u_{\tau} I-\mathbf{z}\right)\left(t-\tau-\left(u_{\tau} I-\mathbf{z}\right)^{-1} y\right)\right.\right. \\
\left.\left.+u_{\tau} C_{4}(t-\tau)+g(t-\tau)\right]\right\} d t .
\end{gathered}
$$

We further restrict the interval to the region $S_{M}=\left\{t:\left|t-\tau-\left(u_{\tau} I-\mathbf{z}\right)^{-1} y\right| \leq M u^{-1}\right\}$ for some large $M$. On the set $S_{M}, u g(t-\tau)=o(1)$. Then,

$$
\begin{aligned}
& \int_{|t-\tau|<\lambda_{u}} e^{u_{t}\left[w_{t}+u_{\tau} C(t-\tau)-u+\mu_{\sigma}(\tau)\right]} d t \\
\geq & (1+o(1)) \exp \left\{\left(u_{\tau}+\zeta_{u}\right)\left[w+\frac{1}{2} y\left(u_{\tau} I-\mathbf{z}\right)^{-1} y\right]+o\left(|y|^{2}\right)\right\} \\
& \times \int_{S_{M}} \exp \left\{\left(u_{\tau}+\zeta_{u}\right)\left[-\frac{1}{2}\left(t-\tau-\left(u_{\tau} I-\mathbf{z}\right)^{-1} y\right)^{\top}\left(u_{\tau} I-\mathbf{z}\right)\left(t-\tau-\left(u_{\tau} I-\mathbf{z}\right)^{-1} y\right)\right]\right\} d t \\
\geq & \delta_{0} u^{-d} \exp \left\{\left(u_{\tau}+\zeta_{u}\right)\left[w+\frac{1}{2} y\left(u_{\tau} I-\mathbf{z}\right)^{-1} y\right]+o\left(|y|^{2}\right)\right\} .
\end{aligned}
$$

We insert the above result back to (0.6) and obtain that

$$
\begin{aligned}
& \int_{T} e^{-\frac{1}{2} u_{t}^{2}+\left[w_{t}+u_{\tau} C(t-\tau)\right] u_{t}} d t \\
\geq & \delta_{0}^{-1} u^{-d} e^{u_{\tau}^{2} / 2} \exp \left\{\left(u_{\tau}+\zeta_{u}\right)\left[w+\frac{1}{2} y\left(u_{\tau} I-\mathbf{z}\right)^{-1} y\right]+o\left(|y|^{2}\right)\right\} \\
= & \Theta(1) u^{-d} e^{u_{\tau}^{2} / 2} \exp \left\{\left(u_{\tau}+\zeta_{u}\right) \mathcal{A}^{\prime}+o\left(u^{-\delta_{0} / 8}|y|^{2}\right)+O(|z|+|y|)\right\} .
\end{aligned}
$$

On the set $\mathcal{L}_{1}$, note that

$$
\mathcal{A}^{\prime}=\mathcal{A}_{\tau}+u^{-1-\delta_{0} / 8} o\left(|y|^{2}+|w|\right)+O\left(u^{-1}+u^{-1}|z|+u^{-1}|y|\right) .
$$


Then, we have that

$\int_{T} e^{-\frac{1}{2} u_{t}^{2}+\left[w_{t}+u_{\tau} C(t-\tau)\right] u_{t}} d t=\Theta(1) u^{-d} e^{u_{\tau}^{2} / 2} \exp \left\{\left(u_{\tau}+\zeta_{u}\right) \mathcal{A}_{\tau}+o\left(u^{-\delta_{0} / 8}|y|^{2}\right)+O(|z|+|y|)\right\}$.

Part 3. With the results of Parts 1 and 2, the analysis of this part is completely analogous to Part 3 of the proof of Theorem 3. For $\imath=0$ we have that

$$
\begin{aligned}
& E^{Q}\left[\left(\int_{T} e^{-\frac{1}{2} u_{t}^{2}+\left[w_{t}+u_{\tau} C(t-\tau)\right] u_{t}} d t\right)^{-2} ; \mathcal{I}(T)>b, \mathcal{L}_{2}, \imath=0\right] \\
= & O(1) u^{2 d} E^{Q}\left\{e^{-u_{\tau}^{2}} E_{\imath, \tau}^{Q}\left[e^{-2\left(u_{\tau}+\zeta_{u}\right) \mathcal{A}_{\tau}+o\left(u^{-\delta_{0} / 8}|y|^{2}\right)+O(|z|+|y|)} ; \mathcal{I}(T)>b\right] ;\left|\tau-t_{*}\right|>u^{-1 / 2+\epsilon}, \imath=0\right\} \\
& +O(1) u^{2 d} E^{Q}\left\{e^{-u_{\tau}^{2}} E_{\imath, \tau}^{Q}\left[e^{-2\left(u_{\tau}+\zeta_{u}\right) \mathcal{A}_{\tau}+o\left(u^{-\delta_{0} / 8}|y|^{2}\right)+O(|z|+|y|)} ; \mathcal{I}(T)>b, \mathcal{L}_{2}\right] ;\left|\tau-t_{*}\right| \leq u^{-1 / 2+\epsilon}, \imath=0\right\} .
\end{aligned}
$$

The first term is on the set $\left|\tau-t_{*}\right|>u^{-1 / 2+\epsilon}$ and therefore $e^{-u_{\tau}^{2}} \leq e^{-u_{t_{*}}^{2}-u^{\epsilon}}$. For the second term, on the set $\left|\tau-t_{*}\right| \leq u^{-1 / 2+\epsilon}$, we have when $|y|>u^{\epsilon}$, or $|z|>u^{\epsilon}$, or $\sup _{|t-\tau|<u^{-1+\delta}}\left|z_{t}-z\right|<u^{-\epsilon}$, the inner expectation is (note that $\xi_{u}=o_{p}(1)$ and has exponential moment)

$$
\begin{aligned}
E_{\iota, \tau}^{Q} & {\left[e^{-2\left(u_{\tau}+\zeta_{u}\right) \mathcal{A}_{\tau}+o\left(u^{-\delta_{0} / 8}|y|^{2}\right)+O(|z|+|y|)} ; \mathcal{I}(T)>b, \mathcal{L}_{2}\right] } \\
& \leq E\left[e^{-\sigma \xi_{u}+o\left(u^{-\delta_{0} / 8}|y|^{2}\right)+O(|z|+|y|)} ; \mathcal{L}_{2}\right]=O\left(Q\left(\mathcal{L}_{2}\right)\right)=O(1) e^{-u^{2 \epsilon} / 2} .
\end{aligned}
$$

For the case that $\imath=1$, the handling is similar. Therefore, the overall contribution is

$$
E^{Q}\left[\left(\int_{T} e^{-\frac{1}{2} u_{t}^{2}+\left[w_{t}+u_{\tau} C(t-\tau)\right] u_{t}} d t\right)^{-2} ; \mathcal{I}(T)>b, \mathcal{L}_{2}\right]=o(1) v^{2}(b) .
$$

Thereby, we conclude the proof.

Proof of Lemma 18. The proof of Lemma 18 is analogous to that of Lemma 17. We only show the key steps here.

Consider the set

$$
\mathcal{L}_{3}=\left\{\sup _{t \in T}|f(t)|<M u, \quad \sup _{t \in T}|\partial f(t)|<M^{2} u, \quad \sup _{t \in T}\left|\partial^{2} f(t)\right|<M^{2} u\right\},
$$

where $M$ is some big constant. By the Borel-TIS lemma, we have

$$
E^{Q}\left[\frac{d P}{d Q} ; \beta_{u}(T)>u, \mathcal{L}_{3}^{c}\right]=P\left(\beta_{u}(T)>u, \mathcal{L}_{3}^{c}\right) \leq P\left(\mathcal{L}_{3}^{c}\right)=o(1) v(b) .
$$

For the second moment, we have that

$$
\begin{aligned}
E^{Q}\left[\left(\frac{d P}{d Q}\right)^{2} ; \beta_{u}(T)>u, \mathcal{L}_{3}^{c}\right] & =\left[\frac{d P}{d Q} ; \beta_{u}(T)>u, \mathcal{L}_{3}^{c}\right] \\
& \leq E\left[\left(\int_{T} e^{-\frac{1}{2} u_{t}^{2}+u_{t} f(t)} d t\right)^{-1} ; \mathcal{L}_{3}^{c}\right] \\
& \leq E\left[e^{u^{2}+u \sup |f(t)|} ; \mathcal{L}_{3}^{c}\right] .
\end{aligned}
$$


We can always choose $M$ large enough such that the term is of order $o\left(v^{2}(b)\right)$. On the set $\mathcal{L}_{3}$, let $t_{\text {sup }}$ be the maximum of $f(t)$ that is $f\left(t_{\text {sup }}\right)=\sup _{t \in T} f(t)$. Then, there exists constant $c_{1}$ such that

$$
\begin{aligned}
\int_{T} L R_{2}(t) d t & =\int_{T} e^{-\frac{1}{2} u_{t}^{2}+u_{t} f(t)} d t \\
& \geq \int_{\left|t-t_{\text {sup }}\right|<u^{-2-\epsilon}} e^{-\frac{1}{2} u_{t}^{2}+u_{t} f(t)} d t \geq e^{-\frac{u^{2}+c_{1} u}{2}+u f\left(t_{\text {sup }}\right)} .
\end{aligned}
$$

Now consider the set $\mathcal{L}_{1}$ defined in $(0.3)$, we have

$$
\begin{aligned}
& E^{Q}\left[\left(\frac{d P}{d Q}\right)^{2} ; \beta_{u}(T)>u, \mathcal{L}_{1}^{c} \cap \mathcal{L}_{3}\right] \\
\leq & E^{Q}\left[\left(\rho_{2} \int_{T} \frac{1}{\operatorname{mes}(T)} L R_{2}(t) d t\right)^{-2} ; \beta_{u}(T)>u, \mathcal{L}_{1}^{c} \cap \mathcal{L}_{3}\right] \\
= & \frac{m^{2} s^{2}(T)}{\rho_{2}^{2}} E^{Q}\left[\left(\int_{T} e^{-\frac{1}{2} u_{t}^{2}+u_{t} f(t)} d t\right)^{-2} ; \beta_{u}(T)>u, \mathcal{L}_{1}^{c} \cap \mathcal{L}_{3}\right] .
\end{aligned}
$$

Then, on the set $\mathcal{L}_{3}$, there exists a constant $c_{2}$ such that

$$
\begin{aligned}
(0.8) & \leq \frac{m e s^{2}(T)}{\rho_{2}^{2}} E^{Q}\left[\left(\int_{T} e^{-\frac{1}{2} u_{t}^{2}+u_{t} f(t)} d t\right)^{-2} ; f\left(t_{\text {sup }}\right)>u-c_{2}, \mathcal{L}_{1}^{c} \cap \mathcal{L}_{3}\right] \\
& \leq \frac{m e s^{2}(T)}{\rho_{2}^{2}} E^{Q}\left[\left(e^{-\frac{u^{2}+c_{1} u}{2}+u f\left(t_{\text {sup }}\right)}\right)^{-2} ; f\left(t_{\text {sup }}\right)>u-c_{2}, \mathcal{L}_{1}^{c} \cap \mathcal{L}_{3}\right]
\end{aligned}
$$

where in the last step we plugged in the bound in (0.7). Therefore there exists a constant $c_{3}$ such that

$$
(0.8) \leq \rho_{2}{ }^{-2} e^{-u^{2}+2 c_{3} u \log u} Q\left(\mathcal{L}_{1}^{c} \cap \mathcal{L}_{3}\right) .
$$

Then the Borel-TIS Lemma implies that on the set $\mathcal{L}_{1}^{c}$, there exists a positive constant $\varpi$ such that

$$
E^{Q}\left[\left(\frac{d P}{d Q}\right)^{2} ; \beta_{u}(T)>u, \mathcal{L}_{1}^{c}\right]=o(1) \rho_{2}{ }^{-2} e^{-u^{2}-u^{1+\varpi}}=o(1) v^{2}(b) .
$$

With exactly the same argument, we have that

$$
E^{Q}\left[\frac{d P}{d Q} ; \beta_{u}(T)>u, \mathcal{L}_{1}^{c}\right]=o(1) v(b)
$$

Now on $\mathcal{L}_{1}$, with a similar three-step procedure explored in the proof of Lemma 17, we can obtain that

$$
E^{Q}\left[\left(\frac{d P}{d Q}\right)^{2} ; \beta_{u}(T)>u, \mathcal{L}_{*}^{c}\right]=o(1) v^{2}(b), \quad E^{Q}\left[\frac{d P}{d Q} ; \beta_{u}(T)>u, \mathcal{L}_{*}^{c}\right]=o(1) v(b) .
$$

With a similar derivation as in Theorem 3 , we obtain that

$$
P\left(\beta_{u}(T)>u\right) \sim v(b) .
$$

The detailed derivation of the above asymptotic approximation is omitted. 
Proof of Lemma 20. For $\delta>\epsilon$, we first split the expectation into two parts:

$$
\begin{aligned}
& E\left[\exp \left\{\sigma\left(u-\mu_{\sigma}(\tau)\right) C_{4}\left((u I-\mathbf{z})^{-\frac{1}{2}} S\right)+\sigma R\left((u I-\mathbf{z})^{-\frac{1}{2}} S\right)\right\}\right] \\
= & E\left[\ldots ;|S| \leq u^{\delta}\right]+E\left[\ldots ;|S|>u^{\delta}\right] \\
= & J_{1}+J_{2} .
\end{aligned}
$$

Let $t=(u I-\mathbf{z})^{-\frac{1}{2}} s$. Given that $C(t)$ is a monotone non-increasing function, we have that for all $|s|=O\left(u^{\delta}\right)$

$$
\begin{aligned}
& -\frac{\sigma}{2}\left(s-(u I-\mathbf{z})^{-1 / 2} \tilde{y}\right)^{\top}\left(s-(u I-\mathbf{z})^{-1 / 2} \tilde{y}\right) \\
& +\sigma\left(u-\mu_{\sigma}(\tau)\right) C_{4}\left((u I-\mathbf{z})^{-\frac{1}{2}} s\right)+\sigma R\left((u I-\mathbf{z})^{-\frac{1}{2}} s\right) \\
= & -\frac{\sigma}{2}\left(t-(u I-\mathbf{z})^{-1 / 2} \tilde{y}\right)^{\top}(u I-\mathbf{z})\left(t-(u I-\mathbf{z})^{-1 / 2} \tilde{y}\right)+\sigma\left(u-\mu_{\sigma}(\tau)\right) C_{4}(t)+\sigma R(t) \\
\leq & -\lambda u|t|^{2}=-\lambda s^{2} .
\end{aligned}
$$

Therefore, for $\lambda^{\prime}$ small

$$
J_{2}=O\left(e^{-\lambda^{\prime} u^{2 \delta}}\right)
$$

We now consider the leading term $J_{1}$. On the set that $|S| \leq u^{\delta}$ and $|w|+|y|+|z|=$ $O\left(u^{1 / 2+\epsilon}\right)$, we have that for sufficiently small $\epsilon$ and $\delta$,

$$
\begin{aligned}
& \sigma\left(u-\mu_{\sigma}(\tau)\right) C_{4}\left((u I-\mathbf{z})^{-\frac{1}{2}} S\right)+\sigma R\left((u I-\mathbf{z})^{-\frac{1}{2}} S\right) \\
= & O\left(u^{-1+4 \delta}\right)+O\left(u^{(-1 / 2+\delta)\left(2+\delta_{0}\right)} u^{1 / 2+\epsilon}\right)=o\left(u^{-1 / 2-\delta_{0} / 8}\right) .
\end{aligned}
$$

By using Taylor's expansion twice, we can essentially move the expectation into the exponent and obtain that $\log J_{1}$ equals

$$
E\left[\sigma\left(u-\mu_{\sigma}(\tau)\right) C_{4}\left((u I-\mathbf{z})^{-\frac{1}{2}} S\right)+\sigma R\left((u I-\mathbf{z})^{-\frac{1}{2}} S\right) ;|S| \leq u^{\delta}\right]+o\left(u^{-1-\delta_{0} / 4}\right) .
$$

Note that $(u I-\mathbf{z})^{-1 / 2} \tilde{y}=u^{-1 / 2} \tilde{y}+O\left(u^{-3 / 2}|\tilde{y}||z|\right)$ and $y=\tilde{y}+O(1)$. Let $Z=\left(Z_{1}, \ldots, Z_{d}\right)$ be a multivariate Gaussian random vector with mean zero and covariance function $\sigma^{-1} I$. Then, $S$ is equal in distribution to $Z+u^{-1 / 2} \tilde{y}+O\left(u^{-3 / 2}|\tilde{y}||z|\right)$. Therefore, for sufficiently small $\epsilon$ and $\delta$, we obtain that

$$
\begin{aligned}
& \log J_{1} \\
= & \frac{\sigma+O\left(u^{-1+\epsilon}\right)}{24 u} \sum_{i j k l} \partial_{i j k l}^{4} C(0) E\left[\left(u^{-1 / 2} \tilde{y}_{i}+Z_{i}\right)\left(u^{-1 / 2} \tilde{y}_{j}+Z_{j}\right)\left(u^{-1 / 2} \tilde{y}_{k}+Z_{k}\right)\left(u^{-1 / 2} \tilde{y}_{l}+Z_{l}\right)\right] \\
& +u^{-1-\delta_{0} / 4} o(|w|+|y|+|z|+1) \\
= & \frac{\sigma}{24} u^{-1} \sum_{i j k l} \partial_{i j k l}^{4} C(0) E\left[\left(u^{-1 / 2} \tilde{y}_{i}+Z_{i}\right)\left(u^{-1 / 2} \tilde{y}_{j}+Z_{j}\right)\left(u^{-1 / 2} \tilde{y}_{k}+Z_{k}\right)\left(u^{-1 / 2} \tilde{y}_{l}+Z_{l}\right)\right] \\
& +u^{-1-\delta_{0} / 4} o(|w|+|y|+|z|+1),
\end{aligned}
$$

where the expectations are taken with respect to $Z$. Then

$$
\begin{aligned}
& \log J_{1} \\
= & \frac{1}{24} u^{-1} \sum_{i i k l} 6 \partial_{i i k l}^{4} C(0) u^{-1} \tilde{y}_{k} \tilde{y}_{l}+\frac{1}{8 \sigma u} \sum_{i i i i} \partial_{i i i i}^{4} C(0)+u^{-1-\delta_{0} / 4} o(|w|+|y|+|z|+1) \\
= & \frac{1}{8 \sigma u} \sum_{i i i i} \partial_{i i i i}^{4} C(0)+u^{-1-\delta_{0} / 4} o(|w|+|y|+|z|+1) .
\end{aligned}
$$


Proof OF Lemma 22. This proof only consists of elementary algebra. We expand the exponent in the second row and the second term in the third row of (4.21). Furthermore, we move the first term in the third row to the last row and move the last term in the fourth row up to the third row. Then, we obtain that $\mathcal{I}_{2}$ equals

$$
\begin{aligned}
& \int_{A^{*},|t-\tau|<u^{-1+\delta^{\prime}}} \exp \left\{\frac{u_{t_{*}}\left(t-t_{*}\right)^{\top} \Delta \mu_{\sigma}\left(t_{*}\right)\left(t-t_{*}\right)}{2}\right\} \times u_{t} \\
& \times \exp \left\{-\lambda u_{t} w_{t}+\lambda u_{t}\left(u_{t}-u_{\tau} C(t-\tau)\right)+(1-\lambda) \frac{\mathbf{1}^{\top}\left(z_{t}-\mu_{02} u_{t}+\mu_{2}(t-\tau) u_{\tau}\right)}{2 \sigma}-\lambda B_{t}-\frac{\mathbf{1}^{\top} \mu_{22} \mathbf{1}}{8 \sigma^{2}}\right\} \\
& \times \exp \left\{\frac{-\left(z_{t}-\mu_{02} u_{t}+\mu_{2}(t-\tau) u_{\tau}\right)^{\top} \mu_{22}^{-1}\left(z_{t}-\mu_{02} u_{t}+\mu_{2}(t-\tau) u_{\tau}\right)}{2}\right. \\
& \left.\quad+\frac{\left(z_{t}+\mu_{2}(t-\tau) u_{\tau}\right)^{\top} \mu_{22}^{-1}\left(z_{t}+\mu_{2}(t-\tau) u_{\tau}\right)}{2}\right\} \\
& \times \exp \left\{\frac{\left(w_{t}+u_{\tau} C(t-\tau)-\mu_{20} \mu_{22}^{-1}\left(z_{t}+\mu_{2}(t-\tau) u_{\tau}\right)\right)^{2}}{2\left(1-\mu_{20} \mu_{22}^{-1} \mu_{02}\right)}-\frac{\left|\mu_{20} \mu_{22}^{-1}\left(z_{t}-\mu_{02} u_{t}+\mu_{2}(t-\tau) u_{\tau}\right)\right|^{2}}{2\left(1-\mu_{20} \mu_{22}^{-1} \mu_{02}\right)}\right\} d t
\end{aligned}
$$

We now work on the exponents in the third and the last row. In particular, we have that

$$
\begin{aligned}
& \frac{-\left(z_{t}-\mu_{02} u_{t}+\mu_{2}(t-\tau) u_{\tau}\right)^{\top} \mu_{22}^{-1}\left(z_{t}-\mu_{02} u_{t}+\mu_{2}(t-\tau) u_{\tau}\right)}{2}+\frac{\left(z_{t}+\mu_{2}(t-\tau) u_{\tau}\right)^{\top} \mu_{22}^{-1}\left(z_{t}+\mu_{2}(t-\tau) u_{\tau}\right)}{2} \\
& +\frac{\left(w_{t}+u_{\tau} C(t-\tau)-\mu_{20} \mu_{22}^{-1}\left(z_{t}+\mu_{2}(t-\tau) u_{\tau}\right)\right)^{2}}{2\left(1-\mu_{20} \mu_{22}^{-1} \mu_{02}\right)}-\frac{\left|\mu_{20} \mu_{22}^{-1}\left(z_{t}-\mu_{02} u_{t}+\mu_{2}(t-\tau) u_{\tau}\right)\right|^{2}}{2\left(1-\mu_{20} \mu_{22}^{-1} \mu_{02}\right)} \\
= & \frac{\mu_{20} \mu_{22}^{-1} \mu_{02} u_{t}^{2}}{2}+u_{t} \mu_{20} \mu_{22}^{-1}\left(z_{t}-\mu_{02} u_{t}+\mu_{2}(t-\tau) u_{\tau}\right) \\
& +\frac{\left(w_{t}+u_{\tau} C(t-\tau)-\mu_{20} \mu_{22}^{-1} \mu_{02} u_{t}\right)^{2}}{2\left(1-\mu_{20} \mu_{22}^{-1} \mu_{02}\right)} \\
& -\frac{2\left(w_{t}+u_{\tau} C(t-\tau)-\mu_{20} \mu_{22}^{-1} \mu_{02} u_{t}\right) \mu_{20} \mu_{22}^{-1}\left(z_{t}-\mu_{02} u_{t}+\mu_{2}(t-\tau) u_{\tau}\right)}{2\left(1-\mu_{20} \mu_{22}^{-1} \mu_{02}\right)} .
\end{aligned}
$$

We further expand the last two rows and obtain that

$$
\begin{aligned}
& \frac{1}{2} u_{t}^{2}+u_{t} w_{t}+u_{t}\left(u_{\tau} C(t-\tau)-u_{t}\right) \\
+ & \frac{\left(w_{t}+u_{\tau} C(t-\tau)-u_{t}\right)^{2}-2\left(w_{t}+u_{\tau} C(t-\tau)-u_{t}\right) \mu_{20} \mu_{22}^{-1}\left(z_{t}-\mu_{02} u_{t}+\mu_{2}(t-\tau) u_{\tau}\right)}{2\left(1-\mu_{20} \mu_{22}^{-1} \mu_{02}\right)} .
\end{aligned}
$$

We put it back to the expression of $\mathcal{I}_{2}$ and obtain that $\mathcal{I}_{2}$ equals

$$
\begin{aligned}
& \int_{A^{*},|t-\tau|<u^{-1+\delta^{\prime}}} \exp \left\{\frac{u_{t_{*}}\left(t-t_{*}\right)^{\top} \Delta \mu_{\sigma}\left(t_{*}\right)\left(t-t_{*}\right)}{2}+\frac{u_{t}^{2}}{2}\right\} \times u_{t} \\
& \times \exp \left\{(1-\lambda) u_{t}\left[w_{t}+u_{\tau} C(t-\tau)-u_{t}\right]+(1-\lambda) \frac{\mathbf{1}^{\top}\left(z_{t}-\mu_{02} u_{t}+\mu_{2}(t-\tau) u_{\tau}\right)}{2 \sigma}-\lambda B_{t}-\frac{\mathbf{1}^{\top} \mu_{22} \mathbf{1}}{8 \sigma^{2}}\right\} \\
& \times \exp \left\{\frac{\left(w_{t}+u_{\tau} C(t-\tau)-u_{t}\right)^{2}-2\left(w_{t}+u_{\tau} C(t-\tau)-u_{t}\right) \mu_{20} \mu_{22}^{-1}\left(z_{t}-\mu_{02} u_{t}+\mu_{2}(t-\tau) u_{\tau}\right)}{2\left(1-\mu_{20} \mu_{22}^{-1} \mu_{02}\right)}\right\} d t .
\end{aligned}
$$


Proof of Lemma 23. We now consider a particular $t \in A^{*} \cap\left\{t:|t-\tau|<u^{-1+\delta^{\prime}}\right\}$. On the localization set $\mathcal{L}$ and $|t-\tau|<u^{-1+\delta^{\prime}}$, we have that

$$
\left|z_{t}-z\right|=O\left(u^{-\epsilon}\right), \quad\left|z_{t}\right|=O\left(u^{\epsilon}\right), \quad\left|y_{t}-y\right|=O\left(u^{-1+\delta^{\prime}+\epsilon}\right) .
$$

In what follows, we show that, for all $t \in\left\{t:|t-\tau|<u^{-1+\delta^{\prime}}\right\}, \mathcal{A}_{\tau}>0$ implies $t \in A^{*}$. On the set $\left\{t:|t-\tau|<u^{-1+\delta^{\prime}}\right\}$ and the set $\mathcal{L}$, by definition, $t \in A^{*}$ if

$w_{t}+u_{\tau} C(t-\tau)-u_{t}+\frac{\left|y_{t}+u_{\tau} \partial C(t-\tau)\right|^{2}}{2 u_{t}}+\frac{\mathbf{1}^{\top}\left(z_{t}-\mu_{02} u_{t}+\mu_{2}(t-\tau) u_{\tau}\right)}{2 \sigma u_{t}}+\frac{B_{t}}{u_{t}}>-\eta u_{t}^{-1}$.

We insert the expansions of $f_{*}(t), C(t-\tau)$ and $\partial C(t-\tau)$ into the above inequality and obtain that

$$
\begin{aligned}
& w+y^{\top}(t-\tau)+\frac{(t-\tau)^{\top} \mathbf{z}(t-\tau)}{2}+R_{f}(t-\tau)+g(t-\tau)-\frac{u_{\tau}|t-\tau|^{2}}{2}+u_{\tau} C_{4}(t-\tau) \\
& +u_{\tau} R_{C}(t-\tau)-\mu_{\sigma}(\tau)+\mu_{\sigma}(t)+\frac{\left|y_{t}\right|^{2}-2 y_{t} u_{\tau}(t-\tau)+u_{\tau}^{2}|t-\tau|^{2}+O\left(|t-\tau|^{3} u_{\tau} y_{t}\right)}{2 u_{t}} \\
& +\frac{\mathbf{1}^{\top}\left(z_{t}-\mu_{02} u_{t}+\mu_{2}(t-\tau) u_{\tau}\right)}{2 \sigma u_{t}}+\frac{B_{t}}{u_{t}}>-\eta u_{t}^{-1} .
\end{aligned}
$$

We further insert $\mathcal{A}_{\tau}$ in and obtain that

$$
\begin{aligned}
& \mathcal{A}_{\tau}+\left(y-\frac{u_{\tau} y_{t}}{u_{t}}\right)^{\top}(t-\tau)+\left(\frac{u_{\tau}^{2}}{2 u_{t}}-\frac{u_{\tau}}{2}\right)|t-\tau|^{2}+\frac{\left|y_{t}\right|^{2}}{2 u_{t}}-\frac{|y|^{2}}{2 u_{\tau}}+\frac{O\left(|t-\tau|^{3} u_{\tau} y_{t}\right)}{2 u_{t}} \\
& +\frac{B_{t}}{u_{t}}-\frac{B_{\tau}}{u_{\tau}}+\frac{\mathbf{1}^{\top}\left(z_{t}-\mu_{02} u_{t}+\mu_{2}(t) u_{\tau}\right)}{2 \sigma u_{t}}-\frac{\mathbf{1}^{\top} z}{2 \sigma u_{\tau}}-\mu_{\sigma}(\tau)+\mu_{\sigma}(t) \\
& +\frac{1}{2}(t-\tau)^{\top} \mathbf{z}(t-\tau)+R_{f}(t-\tau)+g(t-\tau)+u_{\tau} C_{4}(t-\tau)+u_{\tau} R_{C}(t-\tau) \\
& >-\eta u_{t}^{-1}
\end{aligned}
$$

Thus, on the set $\mathcal{L} \cap\left\{t:|t-\tau|<u^{-1+\delta^{\prime}}\right\},(0.9)$ implies that $t \in A^{*}$ if

$$
\mathcal{A}_{\tau}+\eta u^{-1}>-g(t-\tau)+O\left(u^{-1-\epsilon}\right) \text {. }
$$

In addition, $\sup _{|t-\tau|<u^{-1+\delta^{\prime}}}|g(t)|<u^{-1-\delta}$. Thus, for all $\left\{t:|t-\tau|<u^{-1+\delta^{\prime}}\right\}$,

$$
t \in A^{*} \Leftrightarrow \mathcal{A}_{\tau}>-\eta u_{t}^{-1}+O\left(u^{-1-\epsilon}\right) .
$$

Note that $\eta \sim 1 / \log u$. Thus, we have

$$
\mathcal{A}_{\tau}>0 \Rightarrow\left\{t:|t-\tau|<u^{-1+\delta^{\prime}}\right\} \subset A^{*} .
$$

Proof of Lemma 24. Note that $\delta^{*}$ can be chosen arbitrarily small so that $-2 u^{-\delta_{0} / 8}<$ $x<0$ implies $e^{u^{\delta^{*}} x} \geq 1 / 2$. Thus, on the set $-2 u^{-\delta_{0} / 8}<x<0$, we have

$$
\begin{aligned}
& E\left[\frac{1}{\rho_{1}^{2} \kappa_{1,2}^{2}} ; x>\left(1+o\left(u^{-1-\delta_{0} / 4}\right)\right)\left[\xi_{u}+o\left(u^{-\delta_{0} / 8}\right)\right] \mid \imath, \tau, w, y, z, C_{1}\right] \\
\leq & E\left[\frac{1}{\rho_{1}^{2} \kappa_{1,2}^{2}} \mid \imath, \tau, w, y, z, C_{1}\right]=O(1) \rho_{1}^{-2} e^{u^{\delta^{*}}}{ }^{x}
\end{aligned}
$$


and

$$
\begin{aligned}
& E\left[\frac{1}{\left(1-\rho_{1}-\rho_{2}\right)^{2} \kappa_{2,1}^{2}} ; x>\left(1+o\left(u^{-1-\delta_{0} / 4}\right)\right)\left[\xi_{u}+o\left(u^{-\delta_{0} / 8}\right)\right] \mid \imath, \tau, w, y, z, C_{2}\right] \\
\leq & E\left[\frac{1}{\left(1-\rho_{1}-\rho_{2}\right)^{2} \kappa_{2,1}^{2}} \mid \imath, \tau, w, y, z, C_{2}\right]=O(1)\left(1-\rho_{1}-\rho_{2}\right)^{-2}(1-\lambda)^{-d} e^{u^{\delta^{*}}}{ }_{x} .
\end{aligned}
$$

We now proceed to the case $x<-2 u^{-\delta_{0} / 8}$. Under this setting, we have that $x>(1+$ $\left.o\left(u^{-1-\delta_{0} / 4}\right)\right)\left[\xi_{u}+o\left(u^{-\delta_{0} / 8}\right)\right]$ implies $x / 2>\xi_{u}$. Thus, it is sufficient to provide bounds for $E\left[\frac{1}{\rho_{1}^{2} \kappa_{1,2}^{2}} ; x>\xi_{u} \mid \imath, \tau, w, y, z, C_{1}\right], \quad$ and $\quad E\left[\frac{1}{\left(1-\rho_{1}-\rho_{2}\right)^{2} \kappa_{2,1}^{2}} ; x>\xi_{u} \mid \imath, \tau, w, y, z, C_{2}\right]$.

To simplify the notation, let $\tau=0$. For other values of $\tau$, the derivation is completely analogous. We use the notation $E_{w, y, z, C_{i}}[\cdot]$ to denote $E\left[\cdot \mid w, y, z, C_{i}\right]$ and $P_{w, y, z}$ to denote the conditional probability given $w, y, z$.

Note that

$$
e^{-\xi_{u} / u}=E\left[e^{g\left((u I-\mathbf{Z})^{-1 / 2} S\right)}\right]
$$

where $S$ is a random variable with density proportional to (4.17).

The statement of the lemma considers $x<0$. In the proof, we slip the sign and consider $0<x<u^{3 / 2+\epsilon}$. Thus, we have that

$$
\left\{-x>\xi_{u}\right\}=\left\{A_{1}+A_{2}>e^{x / u}\right\}
$$

where

$$
\begin{aligned}
& A_{1}=E\left[e^{g\left((u I-\mathbf{z})^{-1 / 2} S\right)} ;|S| \leq u^{\delta}\right] \\
& A_{2}=E\left[e^{g\left((u I-\mathbf{Z})^{-1 / 2} S\right)} ;|S|>u^{\delta},(u I-\mathbf{z})^{-1 / 2} S \in T\right] .
\end{aligned}
$$

Furthermore, we have that

$$
\log A_{1} \leq \sup _{|s| \leq u^{-1 / 2+\delta}} g(s),
$$

and by the Borell-TIS inequality (Lemma 16), there exists $\lambda^{\prime}, \delta^{*}>0$ such that

$$
P\left(u \cdot \sup _{|s| \leq u^{-1 / 2+\delta}} g(s)>x\right) \leq e^{-\lambda^{\prime} u^{\delta^{*}} x^{2}} .
$$

In addition, on the set $\mathcal{L}$, the process $g$ is localized and

$$
\kappa_{1,2}^{-2}=e^{O\left(u \cdot \sup _{|s| \leq u-1+\delta}|g(s)|\right)}=e^{O\left(u^{-\delta}\right)} .
$$

Therefore, for $c>0$ there exists $\lambda^{\prime \prime}>0$ such that

$$
\begin{aligned}
& E_{w, y, z, C_{1}}\left[\kappa_{1,2}^{-2} ; A_{1}+A_{2}>e^{x / u}, 0<A_{2}<e^{-c u^{2 \delta}}\right] \\
\leq & E_{w, y, z, C_{1}}\left[e^{O\left(u \cdot \sup _{|s| \leq u}-1+\delta|g(s)|\right)} ; u \cdot \sup _{|s| \leq u-1 / 2+\delta} g(s)+O\left(u \cdot e^{-c u^{2 \delta}}\right)>x\right] \\
\leq & e^{-\lambda^{\prime \prime} u^{\delta^{*}} x^{2}} .
\end{aligned}
$$


Similarly, since

$$
\kappa_{2,1}^{-2}=\Theta(1)(1-\lambda)^{-d} e^{O\left(u \cdot \sup _{|s| \leq u-1+\delta}|g(s)|\right)}=\Theta(1)(1-\lambda)^{-d} e^{O\left(u^{-\delta}\right)},
$$

we have

$$
E_{w, y, z, C_{2}}\left[\kappa_{2,1}^{-2} ; A_{1}+A_{2}>e^{x / u}, 0<A_{2}<e^{-c u^{2 \delta}}\right]=O(1)(1-\lambda)^{-d} e^{-\lambda^{\prime \prime} u^{\delta^{*}} x^{2}} .
$$

For the remainder terms, consider $C_{1}$ first

$$
\begin{aligned}
& E_{w, y, z, C_{1}}\left[\kappa_{1,2}^{-2} ; A_{1}+A_{2}>e^{x / u}, A_{2} \geq e^{-c u^{2 \delta}}\right] \\
\leq & E_{w, y, z, C_{1}}\left[\kappa_{1,2}^{-2} ; A_{2} \geq e^{-c u^{2 \delta}}\right] \\
\leq & E_{w, y, z, C_{1}}\left[\kappa_{1,2}^{-2} ; \sup _{u^{\delta} \leq s \leq \sqrt{u}} g(s / \sqrt{u})-\frac{1}{2} s^{2}>-c u^{2 \delta}\right] .
\end{aligned}
$$

Since for all $0<x<u^{3 / 2+\epsilon}$ there exists $\lambda^{\prime}$ sufficiently small such that

$$
\begin{aligned}
P_{w, y, z}\left(A_{2}>e^{-c u^{2 \delta}}\right) & \leq P_{w, y, z}\left(\sup _{u^{\delta} \leq s \leq \sqrt{u}} g(s / \sqrt{u})-\frac{1}{2} s^{2}>-c u^{2 \delta}\right) \\
& \leq e^{-\lambda^{\prime} u^{2}} \leq e^{-\lambda^{\prime} u^{1 / 2-\epsilon} x}
\end{aligned}
$$

therefore

$$
\begin{aligned}
& \leq e^{-\lambda^{\prime} u^{2}} E_{w, y, z, C_{1}}\left[\left.\kappa_{1,2}^{-2}\right|_{u^{\delta} \leq s \leq \sqrt{u}} g(s / \sqrt{u})-\frac{1}{2} s^{2}>-c u^{2 \delta}\right] \\
& =e^{-\lambda^{\prime} u^{2}} E_{w, y, z, C_{1}}\left[\left.e^{O\left(u \cdot \sup _{|s| \leq u}-1+\delta|g(s)|\right)}\right|_{s \in T,|s|>u^{-1 / 2+\delta}} g(s)-\frac{u}{2} s^{2}>-c u^{2 \delta}\right] \\
& =e^{-\lambda^{\prime} u^{2}} e^{O\left(u^{-\delta}\right)} \leq e^{-u^{\delta^{*}} x} .
\end{aligned}
$$

Similarly, for $C_{2}$ is true, by (0.11), the following bound holds.

$$
\begin{aligned}
& E_{w, y, z, C_{2}}\left[\kappa_{2,1}^{-2} ; A_{1}+A_{2}>e^{x / u}, A_{2} \geq e^{-c u^{2 \delta}}\right] \\
\leq & e^{-\lambda^{\prime} u^{2}} E_{w, y, z, C_{2}}\left[\left.\kappa_{2,1}^{-2}\right|_{s \in T,|s|>u^{-1 / 2+\delta}} g(s)-\frac{u}{2} s^{2}>-c u^{2 \delta}\right] \\
= & O(1)(1-\lambda)^{-d} e^{-\lambda^{\prime} u^{2}}=O(1)(1-\lambda)^{-d} e^{-u^{\delta^{*}} x} .
\end{aligned}
$$

Combining the above results, we have the conclusion.

\section{References.}

Liu, J. and G. Xu (2012). Some asymptotic results of gaussian random fields with varying mean functions and the associated processes. Annals of Statistics 40, 262-293.

JINGCHEN LIU

Department OF Statistics,

COLUMBia University,

1255 Amsterdam, New York, NY

E-MAIL: jcliu@stat.columbia.edu
GongJun Xu

School of Statistics,

University of Minnesota, Twin Cities

367 Ford Hall, 224 Church St SE, Minneapolis, MN 55455.

E-MAIL: xuxxx360@umn.edu 